\title{
SEVEN-DIMENSIONAL CONSIDERATIONS OF EINSTEIN'S CONNECTION. I. THE RECURRENCE RELATIONS OF THE FIRST KIND IN $n$-g-UFT
}

\author{
MI AE KIM, KEUM SOOK SO, CHUNG HYUN CHO, \\ and KYUNG TAE CHUNG
}

Received 12 April 2001 and in revised form 27 September 2001

\begin{abstract}
Einstein's connection in a generalized Riemannian manifold $X_{n}$ has been investigated by many authors for lower-dimensional cases $n=2, \ldots, 6$. In a series of papers, we obtain a surveyable tensorial representation of 7-dimensional Einstein's connection in terms of unified field tensor, with main emphasis on the derivation of powerful and useful recurrence relations which hold in $X_{7}$. In this paper, we give a brief survey of Einstein's unified field theory and derive the recurrence relations of the first kind which hold in a general $X_{n}$. All considerations in this paper are restricted to the first and second classes only since the case of the third class, the simplest case, was already studied by many authors.
\end{abstract}

2000 Mathematics Subject Classification: 83E50, 83C05, 58A05.

1. Introduction. In Appendix II to his last book, Einstein [16] proposed a new unified field theory that would include both gravitation and electromagnetism. Although the intent of this theory is physical, its exposition is mainly geometrical. Characterizing Einstein's unified field theory as a set of geometrical postulates in the space time $X_{4}$, Hlavatý [17] gave its mathematical foundation for the first time. Since then Hlavatý and number of mathematicians contributed for the development of this theory and obtained many geometrical consequences of these postulates.

Generalizing $X_{4}$ to $n$-dimensional generalized Riemannian manifold $X_{n}, n$ dimensional generalization of this theory, so called Einstein's $n$-dimensional unified field theory ( $n$ - $g$-UFT hereafter), had been investigated by Wrede [20] and Mishra [19]. On the other hand, corresponding to $n$ - $g$-UFT, Chung [1] introduced a new unified field theory, called the Einstein's $n$-dimensional ${ }^{*} g$-unified field theory $\left(n^{*} \boldsymbol{g}\right.$-UFT hereafter). This theory is more useful than $n$ - $g$-UFT in some physical aspects. Chung et al. obtained many results concerning this theory $[2,5,6,7,8,12,13]$, particularly, proving that $n^{*} g$-UFT is equivalent to $n-g$-UFT so far as the classes and indices of inertia are concerned [3]. The case of the third class, which is the simplest case of both unified field theories, was completely studied for a general $n$ by many authors (e.g., $[8,19,20]$ ). However, in the cases of the first and second classes of both $n$-dimensional 
generalizations, it has not been possible yet to represent the general $n$-dimensional Einstein's connection in a surveyable tensorial form in terms of the unified field tensor $g_{\lambda \mu}$. This is probably due to the complexity of the higher dimensions.

However, the lower-dimensional cases of the Einstein's connection in $n-g$ UFT were investigated by many authors, 2-dimensional case by Jakubowicz [18] and Chung et al. [4], 3-dimensional case by Chung et al. [9, 10, 11], and 4-dimensional case by Hlavatý [17] and many other geometricians. Recently, Chung et al. also studied the Einstein's connection in 4 - $^{*} g$-UFT [1], in 3-and 5 -* $g$-UFT [8], and in 6- $g$-UFT $[14,15]$, and obtained respective Einstein's connection in a surveyable tensorial form.

The purpose of the present paper, the first part of a series of three papers, is to introduce a brief survey of Einstein's $n$ - $g$-UFT and derive several recurrence relations of the first kind which hold in $n-g$-UFT. In the second and third parts, we derive powerful and useful recurrence relations of the second and third kind which hold in 7-g-UFT, prove a necessary and sufficient condition for the existence and uniqueness of the 7-dimensional Einstein's connection, establish a linear system of 66 equations for the solution of the 7-dimensional Einstein's connection employing the powerful recurrence relations, and finally solve the system. We hope that the results obtained in this series of papers, especially the 7-dimensional recurrence relations of the second and third kind in part II, will contribute to the study of generalizing the corresponding results in a general $n$-dimensional case when $n$ is odd.

All considerations in this and subsequent papers are dealt with for the first and second classes only.

2. Preliminaries. This section is a brief collection of basic concepts, notations, and results, which are needed in our subsequent considerations. They are due to Chung [1, 8], Hlavatý [17], and Mishra [19]. All considerations in this section are dealt with for a general $n>1$.

2.1. $n$-dimensional $g$-unified field theory. The Einstein's $n$-dimensional unified field theory, denoted by $n$ - $g$-UFT, is an $n$-dimensional generalization of the usual Einstein's 4-dimensional unified field theory in the space-time $X_{4}$. It is based on the following three principles as indicated by Hlavatý [17].

Principle 2.1. Let $X_{n}$ be an $n$-dimensional generalized Riemannian manifold referred to a real coordinate system $x^{v}$, which obeys the coordinate transformation $x^{v} \rightarrow x^{v^{\prime}}$ (throughout the present paper, Greek indices are used for the holonomic components of tensors, while Roman indices are used for the nonholonomic components of a tensor in $X_{n}$. All indices take the values $1,2, \ldots, n$ and follow the summation convention with the exception of nonholonomic indices $x, y, z, t$ ) for which

$$
\operatorname{det}\left(\frac{\partial x^{\prime}}{\partial x}\right) \neq 0
$$


In $n$ - $g$-UFT, the manifold $X_{n}$ is endowed with a real nonsymmetric tensor $g_{\lambda \mu}$, called the unified field tensor of $X_{n}$. This tensor may be decomposed into its symmetric part $h_{\lambda \mu}$ and skew-symmetric part $k_{\lambda \mu}$,

$$
g_{\lambda \mu}=h_{\lambda \mu}+k_{\lambda \mu}
$$

For the case of $n=4$, the gravitational field is described by 10 components of a symmetric quadratic tensor, while the electromagnetic field is defined by a quadratic skew-symmetric tensor described by 6 components. Therefore, we may expect that a unified theory comprising gravitation and electromagnetism to be based on 16 field quantities. Several attempts in this direction were made by Einstein and ultimately discarded. As given in (2.2), his last attempt, which he regarded as final, was to introduce the unified field by a real nonsymmetric tensor $g_{\lambda \mu}$, the gravitational field by its symmetric part $h_{\lambda \mu}$, and the electromagnetic field by a skew-symmetric quadratic tensor $k_{\lambda \mu}$ which may be represented as a linear combination of its skew-symmetric part $k_{\lambda \mu}$, where

$$
\mathfrak{g}=\operatorname{det}\left(g_{\lambda \mu}\right) \neq 0, \quad \mathfrak{h}=\operatorname{det}\left(h_{\lambda \mu}\right) \neq 0, \quad \mathfrak{k}=\operatorname{det}\left(k_{\lambda \mu}\right) \text {. }
$$

We may define a unique tensor $h^{\lambda v}=h^{\nu \lambda}$ by

$$
h_{\lambda \mu} h^{\lambda \nu}=\delta_{\mu}^{v}
$$

In $n$-g-UFT, the tensors $h_{\lambda \mu}$ and $h^{\lambda v}$ will serve for raising and/or lowering indices of tensors in $X_{n}$ in the usual manner.

PRINCIPLE 2.2. The differential geometric structure on $X_{n}$ is imposed by the tensor $g_{\lambda \mu}$ by means of a connection $\Gamma_{\lambda \mu}^{\nu}$, defined by a system of equations

$$
D_{\omega} g_{\lambda \mu}=2 S_{\omega \mu}^{\alpha} g_{\lambda \alpha}
$$

For 4-dimensional case, the tensor field $g_{\lambda \mu}$ imposes a differential geometric structure on space-time $X_{4}$ through the Einstein's connection $\Gamma_{\lambda \mu}^{\nu}$, which Einstein originally defined by the following system of 64 equations, called Einstein's equations:

$$
\partial_{\omega} g_{\lambda \mu}-\Gamma_{\lambda \omega}^{\alpha} g_{\alpha \mu}-\Gamma_{\omega \mu}^{\alpha} g_{\lambda \alpha}=0
$$

The equivalence of (2.5) and (2.6) was proved by Hlavatý [17, page 50]. This system of 64 equations is similar in structure to the system of 40 equations which defines the Christoffel symbols in the gravitational theory. The only striking difference between these two systems is the position of the indices $\omega \mu$ in $\Gamma_{\omega \mu}^{v}$. However, this peculiar order of the indices $\omega \mu$ has a profound geometrical meaning, which Einstein interpreted in his book, "Generalization of Gravitation Theory.” (A reprint of Appendix II from the fourth edition of the 
Meaning of Relativity), Princeton University Press, 1953, as follows: "this fact is the mathematical expression of the indifference of the theory to the sign of electricity." This is an intuition of genius, since Einstein was not in possession of the tensor $\boldsymbol{k}_{\lambda \mu}$ of Electromagnetism.

Here $D_{\omega}$ denotes the symbolic vector of the covariant derivative with respect to $\Gamma_{\lambda \mu}^{v}$, and $S_{\lambda \mu}{ }^{\nu}=\Gamma_{[\lambda \mu]}^{v}$ is the torsion tensor of $\Gamma_{\lambda \mu}^{v}$. The connection $\Gamma_{\lambda \mu}^{v}$ satisfying (2.5) is called the Einstein's connection. Under certain conditions the system (2.5) admits a unique solution $\Gamma_{\lambda \mu}^{\nu}$.

PRINCIPLE 2.3. In order to obtain $g_{\lambda \mu}$ involved in the solution for $\Gamma_{\lambda \mu}^{\nu}$ certain conditions are imposed. These conditions may be condensed to

$$
S_{\lambda}=S_{\lambda \alpha}^{\alpha}=0, \quad R_{[\mu \lambda]}=\partial_{[\mu} X_{\lambda]},
$$

where $X_{\lambda}$ is an arbitrary nonzero vector, and $R_{\omega \mu \lambda}{ }^{v}$ and $R_{\mu \lambda}$ are the curvature tensors of $\Gamma_{\lambda \mu}^{v}$ defined by

$$
\left.R_{\omega \mu \lambda}{ }^{v}=2\left(\partial_{[\mu} \Gamma_{|\lambda|}{ }^{v} \omega\right]+\Gamma_{\alpha}{ }^{v}\left[\mu \Gamma_{|\lambda|}{ }^{\alpha} \omega\right]\right), \quad R_{\mu \lambda}=R_{\alpha \mu \lambda}{ }^{\alpha} .
$$

2.2. Algebraic preliminaries. In this subsection, we introduce notations, concepts, and several algebraic results in $n$-g-UFT.

2.2.1. Notations. The following scalars, tensors, and notations are frequently used in our further considerations:

$$
\begin{aligned}
g & =\frac{\mathfrak{g}}{\mathfrak{h}}, \quad k=\frac{\mathfrak{k}}{\mathfrak{h}}, \\
K_{p} & =k_{\left[\alpha_{1}\right.}{ }^{\alpha_{1}} k_{\alpha_{2}}{ }^{\alpha_{2}} \cdots k_{\left.\alpha_{p}\right]}{ }^{\alpha_{p}}, \quad(p=0,1,2, \ldots), \\
{ }^{(0)} k_{\lambda}{ }^{v} & =\delta_{\lambda}^{v}, \quad{ }^{(1)} k_{\lambda}{ }^{v}=k_{\lambda}{ }^{v}, \quad{ }^{(p)} k_{\lambda}{ }^{v}={ }^{(p-1)} k_{\lambda}{ }^{\alpha} k_{\alpha}{ }^{v}, \quad(p=1,2, \ldots), \\
K_{\omega \mu \nu} & =\nabla_{\nu} k_{\omega \mu}+\nabla_{\omega} k_{v \mu}+\nabla_{\mu} k_{\omega \nu}, \\
\sigma & = \begin{cases}0, & \text { if } n \text { is even, } \\
1, & \text { if } n \text { is odd, }\end{cases}
\end{aligned}
$$

where $\nabla_{\omega}$ is the symbolic vector of covariant derivative with respect to the Christoffel symbols $\left\{\begin{array}{c}v \\ \lambda \mu\end{array}\right\}$ defined by $h_{\lambda \mu}$. It has been shown that the scalars and tensors introduced in (2.9) satisfy

$$
\begin{gathered}
K_{0}=1, \quad K_{n}=k \quad \text { if } n \text { is even, } \quad K_{p}=0 \text { if } p \text { is odd, } \\
g=1+K_{2}+\cdots+K_{n-\sigma}, \\
{ }^{(p)} k_{\lambda \mu}=(-1)^{p(p)} k_{\mu \lambda}, \quad{ }^{(p)} k^{\lambda \nu}=(-1)^{p(p)} k^{\nu \lambda} .
\end{gathered}
$$


Denoting an arbitrary tensor $T_{\omega \mu \lambda}$, skew-symmetric in the first two indices, by $T$, we also use the following useful abbreviations:

$$
\begin{aligned}
& \stackrel{p q r}{T}=\stackrel{p q r}{T} \omega \mu \lambda=T_{\alpha \beta \gamma}{ }^{(p)} k_{\omega}{ }^{\alpha(q)} k_{\mu}{ }^{\beta(r)} k_{\lambda}{ }^{\gamma}, \\
& T=T_{\omega \mu \lambda}=\stackrel{000}{T}, \\
& 2 \stackrel{p q r}{T}_{\omega[\lambda \mu]}=\stackrel{p q r}{T} \omega \lambda \mu-\stackrel{p q r}{T}_{\omega \mu \lambda}, \\
& 2 \stackrel{(p q) r}{T}_{\omega \lambda \mu}=\stackrel{p q r}{T} \omega \lambda \mu+\stackrel{q p r}{T} \omega \lambda \mu, \quad \text { etc. }
\end{aligned}
$$

We then have

$$
\stackrel{p q r}{T} \omega \lambda \mu=-{ }^{q p r} \lambda \lambda \omega \mu
$$

\subsubsection{Classification, basic vectors, and basic scalars}

DEFINITION 2.4. The tensor $g_{\lambda \mu}$ (or $k_{\lambda \mu}$ ) is said to be

(1) of the first class, if $K_{n-\sigma} \neq 0$,

(2) of the second class with the $j$ th category $(j \geq 1)$, if

$$
K_{2 j} \neq 0, \quad K_{2 j+2}=K_{2 j+4}=\cdots=K_{n-\sigma}=0,
$$

(3) of the third class, if $K_{2}=K_{4}=\cdots=K_{n-\sigma}=0$.

The solution of the system of (2.5) is most conveniently brought about in a nonholonomic frame of reference, which may be introduced by the projectivity

$$
M A^{v}=k_{\mu}{ }^{\nu} A^{\mu}, \quad(M \text { a scalar }) .
$$

DEFINITION 2.5. An eigenvector $A^{v}$ of $k_{\lambda \mu}$ satisfying (2.14) is called a basic vector in $X_{n}$, and the corresponding eigenvalue $M$ is termed a basic scalar.

It has been shown that the basic scalars $M$ are solutions of the characteristic equation

$$
M^{\sigma}\left(M^{n-\sigma}+K_{2} M^{n-2-\sigma}+\cdots+K_{n-2-\sigma} M^{2}+K_{n-\sigma}\right)=0 .
$$

2.2.3. Nonholonomic frame of reference. In the first and second classes, we have a set of $n$ linearly independent basic vectors $\underset{i}{A_{i}^{v}}(i=1, \ldots, n)$ and a unique reciprocal set $A_{\lambda}(i=1, \ldots, n)$, satisfying

$$
\stackrel{j}{A_{\lambda}} \underset{i}{A^{\lambda}}=\delta_{i}^{j}, \quad \stackrel{i}{A_{\lambda}} A_{i}^{v}=\delta_{\lambda}^{v} .
$$

With these two sets of vectors, we may construct a nonholonomic frame of reference as follows. 
DEFINITION 2.6. If $T_{\lambda \ldots}^{v \cdots}$ are holonomic components of a tensor, then its nonholonomic components $T_{j \ldots}^{i \cdots}$ are defined by

$$
T_{j \cdots}^{i \cdots}=T_{\lambda \cdots}^{v \cdots} \stackrel{i}{A}_{v} \cdots \underset{j}{A_{j}^{\lambda} \cdots}
$$

An easy inspection shows that

$$
T_{\lambda \cdots}^{v \cdots}=T_{j \cdots}^{i \cdots A_{i}}{ }_{i}^{v} \cdots A_{\lambda}^{j} \cdots
$$

Furthermore, if $\underset{x}{M}$ is the basic scalar corresponding to $\underset{x}{A^{v}}$, then the nonholonomic components of ${ }^{(p)} k_{\lambda}{ }^{v}$ are given by

$$
{ }^{(p)} k_{x}{ }^{i}=M_{x}{ }^{p} \delta_{x}^{i}, \quad{ }^{(p)} k_{x i}={\underset{x}{M}}^{p} h_{x i}, \quad{ }^{(p)} k^{x i}=\underset{x}{M^{p}} h^{x i} .
$$

Without loss of generality we may choose the nonholonomic components of $h_{\lambda \mu}$ as

$$
\begin{aligned}
h_{12} & =h_{34}=\cdots=h_{n-1-\sigma, n-\sigma}=1, \\
\sigma h_{n i_{0}} & =\delta_{\sigma}^{1}, \quad \text { the remaining } h_{i j}=0,
\end{aligned}
$$

where the index $i_{0}$ is taken so that $\operatorname{Det}\left(h_{i j}\right) \neq 0$ when $n$ is odd.

2.3. Differential geometric preliminaries. In this subsection, we present several useful results involving Einstein's connection. These results are needed in our subsequent considerations for the solution of (2.5).

If the system (2.5) admits a solution $\Gamma_{\lambda \mu}^{\nu}$, it must be of the form

$$
\Gamma_{\lambda \mu}^{v}=\left\{\begin{array}{c}
v \\
\lambda \mu
\end{array}\right\}+S_{\lambda \mu}^{v}+U_{\lambda \mu}^{v}
$$

where

$$
U_{v \lambda \mu}=2 \stackrel{001}{S} v(\lambda \mu)
$$

The above two relations show that our problem of determining $\Gamma_{\lambda \mu}^{\nu}$ in terms of $g_{\lambda \mu}$ is reduced to that of studying the tensor $S_{\lambda \mu}{ }^{\nu}$. On the other hand, it has been shown that the tensor $S_{\lambda \mu}{ }^{v}$ satisfies

$$
S=B-3 \stackrel{(110)}{S}
$$

where

$$
2 B_{\omega \mu \nu}=K_{\omega \mu \nu}+3 K_{[\alpha \beta \nu]} k_{\omega}{ }^{\alpha} k_{\mu}{ }^{\beta} .
$$


Therefore, the Einstein's connection $\Gamma_{\lambda \mu}^{\nu}$ satisfying (2.5) may be determined if the solution $S_{\lambda \mu}{ }^{v}$ of the system (2.24) is found. The main purpose of the present paper is to find a device to solve the system (2.24) when $n=7$.

Furthermore, for the first two classes, the nonholonomic solution of (2.24) is given by

$$
\underset{x y z}{M} S_{x y z}=B_{x y z}
$$

or equivalently

$$
2 \underset{x y z}{M} S_{x y z}=K_{x y z}+3 K_{[x y z]} \underset{x}{M} \underset{y}{M}
$$

where

$$
\underset{x y z}{M}=1+\underset{x}{M} \underset{y}{M}+\underset{y}{M} \underset{z}{M}+\underset{z}{M}
$$

Therefore, in virtue of (2.26), we see that a necessary and sufficient nonholonomic condition for the system (2.5) to have a unique solution in the first two classes is

$$
\underset{x y z}{M} \neq 0 \quad \forall x, y, z
$$

3. The recurrence relations of the first kind in $n-g$-UFT. This section is devoted to the derivation of the recurrence relations of the first kind and two other useful relations which hold in $n$ - $g$-UFT. All considerations in this section are also dealt with for a general $n>1$.

The recurrence relations of the first kind in $n$-g-UFT are those which are satisfied by the tensors ${ }^{(p)} k_{\lambda}{ }^{v}$. These relations will be proved in the following theorem.

THEOREM 3.1 (the recurrence relations of the first kind in $n$ - $g$-UFT). The tensors ${ }^{(p)} k_{\lambda}{ }^{v}$ satisfy the following recurrence relations:

For the first class:

$$
{ }^{(n+p)} k_{\lambda}{ }^{v}+K_{2}{ }^{(n+p-2)} k_{\lambda}{ }^{v}+\cdots+K_{n-\sigma-2}{ }^{(p+\sigma+2)} k_{\lambda}{ }^{v}+K_{n-\sigma^{(p+\sigma)}} k_{\lambda}{ }^{v}=0
$$

which may be condensed to

$$
\sum_{f=0}^{n-\sigma} K_{f}^{(n+p-f)} k_{\lambda}^{v}=0, \quad(p=0,1,2, \ldots) .
$$

For the second class with the jth category:

$$
{ }^{(2 j+p)} k_{\lambda}{ }^{v}+K_{2}{ }^{(2 j+p-2)} k_{\lambda}{ }^{v}+\cdots+K_{2 j}{ }^{(p)} k_{\lambda}{ }^{v}=0
$$


which may be condensed to

$$
\sum_{f=0}^{2 j} K_{f}^{(2 j+p-f)} k_{\lambda}^{v}=0, \quad(p=1,2, \ldots) .
$$

\section{PROOF}

THE CASE OF THE FIRST CLASS. Let $\underset{x}{M}$ be a basic scalar. Then, in virtue of (2.15), we have

$$
\sum_{f=0}^{n-\sigma} K_{f} M_{x}^{n-f}=0
$$

Multiplying $\delta_{x}^{i}$ to both sides of (3.3) and making use of (2.19), we have

$$
\sum_{f=0}^{n-\sigma} K_{f}^{(n-f)} k_{x}^{i}=0
$$

whose holonomic form is

$$
\sum_{f=0}^{n-\sigma} K_{f}^{(n-f)} k_{\lambda}^{\alpha}=0
$$

The relation (3.1) immediately follows by multiplying ${ }^{(p)} k_{\alpha}{ }^{v}$ to both sides of (3.5).

THE CASE OF THE SECOND ClASS WITH THE $j$ TH CATEgORY. When $g_{\lambda \mu}$ belongs to the second class with the $j$ th category, the characteristic equation (2.15) is reduced to

$$
\sum_{f=0}^{2 j} K_{f} M^{n-f}=M^{n-2 j} \sum_{f=0}^{2 j} K_{f} M^{2 j-f}=0 .
$$

Hence, if $\underset{x}{M}$ is a root of (3.6), it satisfies

$$
\underset{x}{M} \sum_{f=0}^{2 j} K_{f} M_{x}^{2 j-f}=\sum_{f=0}^{2 j} K_{f} M_{x}^{2 j-f+1}=0
$$

In virtue of (2.19), multiplication of $\delta_{x}^{i}$ to both sides of (3.7) gives

$$
\sum_{f=0}^{2 j} K_{f}^{(2 j-f+1)} k_{x}^{i}=0 .
$$

The holonomic form of (3.8) is

$$
\sum_{f=0}^{2 j} K_{f}^{(2 j-f+1)} k_{\lambda}^{\alpha}=0 .
$$


Consequently, the relation (3.2) follows by multiplying ${ }^{(p-1)} k_{\alpha}{ }^{v}$ to both sides of (3.9).

REMARK 3.2. When $g_{\lambda \mu}$ belongs to the second class with the first category, the relation (3.2), is reduced to

$$
{ }^{(p+2)} k_{\lambda}{ }^{v}+K_{2}{ }^{(p)} k_{\lambda}{ }^{v}=0, \quad(p=1,2, \ldots) .
$$

In the following two theorems we prove two useful relations.

THEOREM 3.3 (for the first and second classes). In the first two classes, a tensor $T_{\omega \mu \nu}$, skew-symmetric in the first two indices, satisfies

$$
\begin{aligned}
& \stackrel{(p q) r}{T}_{\omega \mu \nu}=\sum_{x, y, z} T_{x y z} \underset{x}{M^{(p}} \underset{y}{M^{q)}} \underset{z}{M^{r}} \stackrel{x}{A_{\omega}} \stackrel{y}{A_{\mu} A_{\nu},}, \\
& \stackrel{r(p q)}{T}_{v[\omega \mu]}=\sum_{x, y, z} T_{x[y z]} M_{y}^{(p} M_{z}^{q)} M_{x}{ }^{r} A_{\nu}{ }^{y} A_{\omega}{ }^{z} A_{\mu} .
\end{aligned}
$$

Proof. Making use of (2.18) and (2.20), the relation (3.11) may be proved as in the following way:

$$
\begin{aligned}
& \stackrel{(p q) r}{T} \omega \mu \nu=\sum_{x, y, z} \stackrel{(p q) r}{T} \underset{x y z}{ } \stackrel{x}{A_{\omega}} \quad \stackrel{y}{A_{\mu}} \stackrel{z}{A}_{v}
\end{aligned}
$$

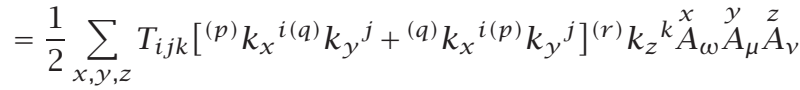

$$
\begin{aligned}
& =\frac{1}{2} \sum_{x, y, z} T_{x y z}\left(\underset{x}{M^{p}} \underset{y}{M^{q}}+\underset{x}{M^{q}} \underset{y}{M^{p}}\right) \underset{z}{M^{r}} \stackrel{x}{A_{\omega}} \underset{A_{\mu}}{y} A_{\nu}^{z} .
\end{aligned}
$$

The second relation can be proved similarly.

Theorem 3.4 (for all classes). The tensor $B_{\omega \mu \nu}$, given by (2.25), satisfies

$$
\begin{aligned}
& \stackrel{(p q) r}{B}=\stackrel{(p q) r}{S}+\stackrel{\left(p^{\prime} q^{\prime}\right) r}{S}+\stackrel{\left(p^{\prime} q\right) r^{\prime}}{S}+\stackrel{\left(p q^{\prime}\right) r^{\prime}}{S}, \\
& 2 \stackrel{(p q) r}{B}_{\omega \mu \nu}=\stackrel{(p q) r}{K}_{\omega \mu \nu}+\stackrel{\left(p^{\prime} q^{\prime}\right) r}{K} \omega \mu \nu+{\stackrel{(p}{\prime} q) r^{\prime}}^{K} v[\omega \mu]+\stackrel{\left(p q^{\prime}\right) r^{\prime}}{K} v[\omega \mu],
\end{aligned}
$$

where

$$
p^{\prime}=p+1, \quad q^{\prime}=q+1, \quad r^{\prime}=r+1 .
$$

Proof. In virtue of (2.11) and (2.24), the relation (3.14) may be shown as in the following way:

$$
\begin{aligned}
\underset{B}{(p q) r}= & \stackrel{(p q) r}{B} \omega \mu \nu=\frac{1}{2} B_{\alpha \beta \gamma}\left[{ }^{(p)} k_{\omega}{ }^{\alpha(q)} k_{\mu}{ }^{\beta}+{ }^{(q)} k_{\omega}{ }^{\alpha(p)} k_{\mu}{ }^{\beta}\right]^{(r)} k_{\nu}{ }^{\gamma} \\
= & \frac{1}{2}\left[S_{\alpha \beta \gamma}+S_{\epsilon \eta \gamma} k_{\alpha}{ }^{\epsilon} k_{\beta}{ }^{\eta}+S_{\epsilon \beta \eta} k_{\alpha}{ }^{\epsilon} k_{\gamma}{ }^{\eta}+S_{\alpha \epsilon \eta} k_{\beta}{ }^{\epsilon} k_{\gamma}{ }^{\eta}\right] \\
& \times\left[{ }^{(p)} k_{\omega}{ }^{\alpha(q)} k_{\mu}{ }^{\beta}+{ }^{(q)} k_{\omega}{ }^{\alpha(p)} k_{\mu}{ }^{\beta}\right]^{(r)} k_{\nu}{ }^{\gamma} .
\end{aligned}
$$


After a lengthy calculation, we note that the right-hand side of the above equation is equal to (3.14). The relation (3.15) may be proved similarly.

ACKNOWLEDGment. This work was partially supported by the Basic Science Research Institute Program, Ministry of Education, Republic of Korea, 1997, BSRI-97-1442.

\section{REFERENCES}

[1] K. T. Chung, Einstein's connection in terms of ${ }^{*} g^{\lambda \mu}$, Nuovo Cimento (10) 27 (1963), 1297-1324.

[2] K. T. Chung and K. S. Chang, Degenerate cases of the Einstein's connection in the ${ }^{*} g^{\lambda v}$-unified field theory. I, Tensor (N.S.) 20 (1969), 143-149.

[3] K. T. Chung and D. H. Cheoi, A study on the relations of two n-dimensional unified field theories, Acta Math. Hungar. 45 (1985), no. 1-2, 141-149.

[4] K. T. Chung and C. H. Cho, Some recurrence relations and Einstein's connection in 2-dimensional unified field theory, Acta Math. Hungar. 41 (1983), no. 1-2, 47-52.

[5] K. T. Chung, P. U. Chung, and I. H. Hwang, The curvature tensors in the Einstein's $* g$-unified field theory. I. The SE-curvature tensor of ${ }^{*} g$-SEX $n$, J. Korean Math. Soc. 35 (1998), no. 4, 1045-1060.

[6] _ _ The curvature tensors in the Einstein's ${ }^{*} g$-unified field theory. II. The contracted SE-curvature tensors of ${ }^{*} g$-SEX $n$, Bull. Korean Math. Soc. 35 (1998), no. 4, 641-652.

[7] K. T. Chung and T. S. Han, $n$-dimensional representations of the unified field tensor ${ }^{*} g^{\lambda v}$, Int. J. Theoret. Phys. 20 (1981), no. 10, 739-747.

[8] K. T. Chung and I. H. Hwang, Three- and five-dimensional considerations of the geometry of Einstein's ${ }^{*} g$-unified field theory, Int. J. Theoret. Phys. 27 (1988), no. 9, 1105-1136.

[9] K. T. Chung and D. K. Jun, On the Einstein's connection of 3-dimensional unified field theory of the third class, J. NSRI(Yonsei University) 7 (1981), 7-12.

[10] K. T. Chung and S. J. Kang, On the Einstein's connection of 3-dimensional unified field theory of the second class, J. NSRI(Yonsei University) 4 (1979), 5-10.

[11] K. T. Chung and J. W. Lee, On the algebra of 3-dimensional unified field theory for the third class, J. NSRI(Yonsei University) 6 (1980), 1-6.

[12] K. T. Chung, M. S. Oh, and J. M. Ko, The submanifolds $X_{m}$ of the manifold ${ }^{*} g$ $\mathrm{MEX}_{n}$. I. The induced connection on $X_{m}$ of ${ }^{*} g-\mathrm{MEX}_{n}$, Note Mat. 18 (1998), no. 2, 213-225.

[13] _ The submanifolds $X_{m}$ of the manifold ${ }^{*} g-\mathrm{MEX}_{n}$. II. Fundamental equations on $X_{m}$ of ${ }^{*} g$-MEX $X_{n}$, Note Mat. 18 (1998), no. 2, 227-234.

[14] K. T. Chung, G. T. Yang, and I. H. Hwang, Six-dimensional considerations of Einstein's connection for the first two classes. I. The recurrence relations in 6-g-UFT, Int. J. Math. Math. Sci. 22 (1999), no. 3, 469-482.

[15] _ Six-dimensional considerations of Einstein's connection for the first two classes. II. The Einstein's connection in 6-g-UFT, Int. J. Math. Math. Sci. 22 (1999), no. 3, 483-488.

[16] A. Einstein, The Meaning of Relativity. Third edition, revised, including The Generalized Theory of Gravitation, Princeton University Press, New Jersey, 1950.

[17] V. Hlavatý, Geometry of Einstein's Unified Field Theory, P. Noordhoff, Groningen, 1957. 
[18] A. Jakubowicz and J. Klekowska, The necessary and sufficient condition for the existence of the unique connection of the two-dimensional generalized Riemann space, Tensor (N.S.) 20 (1969), 72-74.

[19] R. S. Mishra, n-dimensional considerations of unified theory of relativity. Recurrence relations, Tensor (N.S.) 9 (1959), 217-225.

[20] R. C. Wrede, " $n$ " dimensional considerations of basic principles $A$ and $B$ of the unified theory of relativity, Tensor (N.S.) 8 (1958), 95-122.

Mi Ae Kim: Department of Mathematics, Yonsei University, Seoul 120-749, Korea

Keum Sook So: Department of Mathematics, Hallym University, Chunchon 200-702, Korea

E-mail address: ksso@sun.ha17ym.ac.kr

Chung Hyun Cho: Department of Mathematics, Inha University, Inchon 402-751, Korea

E-mail address: chcho@math.inha.ac.kr

Kyung Tae Chung: Department of Mathematics, Yonsei University, Seoul 120-749, Korea

E-mail address: ktchung@yonsei .ac.kr 




Advances in

Operations Research

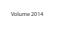



\section{The Scientific} World Journal
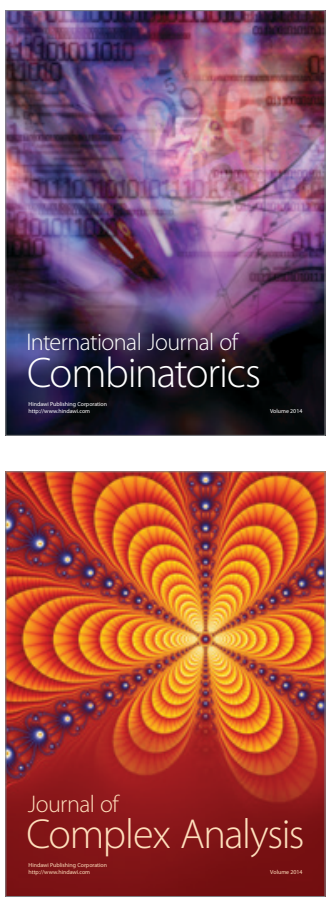

International Journal of

Mathematics and

Mathematical

Sciences
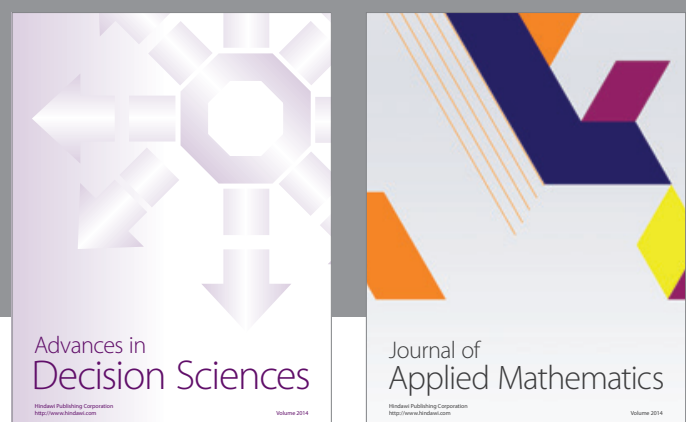

Journal of

Applied Mathematics
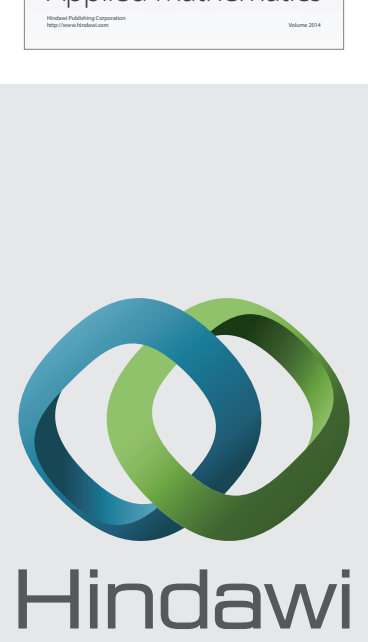

Submit your manuscripts at http://www.hindawi.com


Mathematical Problems in Engineering
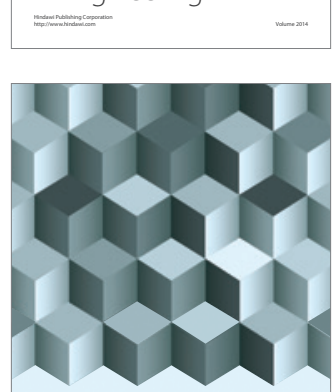

Journal of

Function Spaces


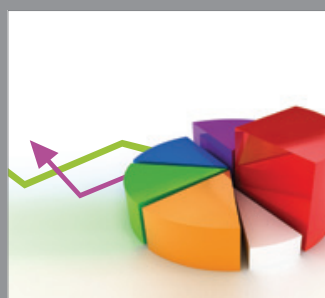

ournal of

Probability and Statistics

Promensencen
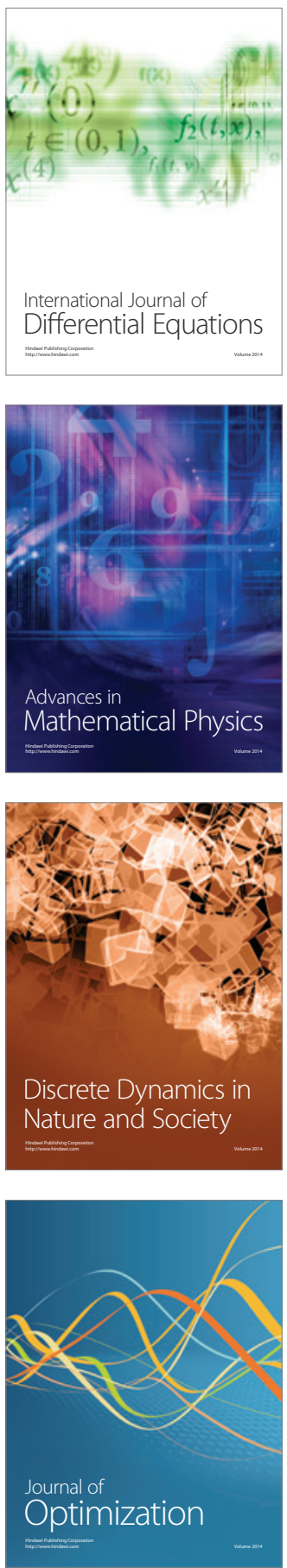